\title{
Functional Identification of Cancer-relevant Genes through Large-Scale RNA Interference Screens in Mammalian Cells
}

\author{
T.R. Brummelkamp, K. Berns, E.M. Hijmans, J. Mullenders, A. Fabius, M. Heimerikx, \\ A. Velds, R.M. Kerkhoven, M. Madiredjo, R. Bernards, And R.L. Beijersbergen \\ Division of Molecular Carcinogenesis and Center for Biomedical Genetics, The Netherlands Cancer Institute, \\ 1066 CX Amsterdam, The Netherlands
}

The discovery of a cellular response against doublestranded RNA (Fire et al. 1998) has provided one of the most powerful tools to manipulate gene expression and this has revolutionized loss-of-function genetics in Caenorhabditis elegans and Drosophila (Ashrafi et al. 2003; Kamath et al. 2003; Lum et al. 2003). In most mammalian cells, however, the introduction of long double-stranded RNA provokes an interferon response, leading to a general shutoff of protein synthesis (Stark et al. 1998). This response can be bypassed by using chemically synthesized, 21-base-pair double-stranded short interfering RNA (siRNAs), which can cause strong, but transient, inhibition of gene expression in nearly all mammalian cells (Elbashir et al. 2001). Indeed, small-scale genetic screens with sets of in vitro synthesized siRNAs have recently been performed in mammalian cells to identify modulators of apoptosis (Aza-Blanc et al. 2003). However, the use of these siRNAs is limited by the transient inhibition of gene expression and their high cost. To circumvent these limitations of siRNAs, we and others have developed expression vectors that direct the synthesis of short hairpin RNAs (shRNAs), which are processed in vivo to siRNA-like molecules that can suppress gene expression over prolonged periods of time (Brummelkamp et al. 2002b; Miyagishi and Taira 2002; Paddison et al. 2002; Sui et al. 2002; Yu et al. 2002). We have recently shown the feasibility of using shRNA vectors to identify loss-of-function phenotypes in mammalian cells by creating a set of vectors to suppress nearly all members of the family of de-ubiquitinating enzymes. Using this approach, we identified the cylindromatosis tumor suppressor gene as a key regulator of the transcription factor NF- $\mathrm{KB}$ (Brummelkamp et al. 2003). More recently, we constructed a large set of shRNA vectors that together target some 8000 human genes for suppression (Berns et al. 2004). We discuss here several ways in which such shRNA vector libraries can be used to identify novel components of cancer-relevant pathways.

\section{A LIBRARY OF ShRNA VECTORS}

We generated a shRNA vector library by first selecting nearly 8000 human genes for shRNA-mediated knockdown. The genes were chosen because they were either components of major cellular pathways including cell cycle, transcription regulation, stress signaling, and signal transduction or were involved in important biological processes such as biosynthesis, proteolysis, and metabolism. In addition, a large set of genes implicated in cancer and other diseases was included in the shRNA library. To increase the likelihood of obtaining a functionally significant inhibition of gene expression, we constructed three different shRNA vectors against each gene transcript (Fig. 1A). Thus, the shRNA library consists in total of some 24,000 shRNA vectors. To construct the library, we first selected three 19-mer sequences from each transcript and incorporated each of these 19-mers into two complementary 59-mer DNA oligonucleotides (Fig. 1A). The design of the two 59-mer oligonucleotides was such that, after transcription, the pRETRO-SUPER-encoded RNA transcript will have the form of a short hairpin, in which the two self-complementary stretches in the

A.

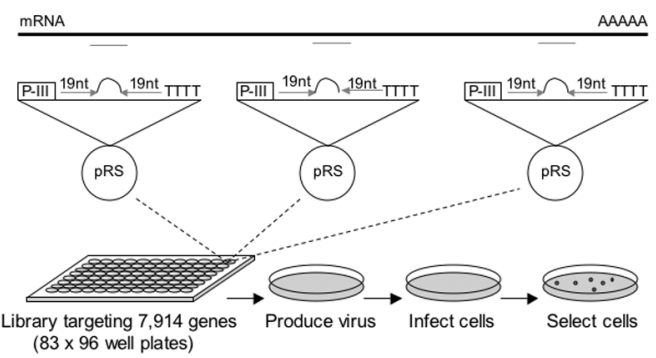

B.

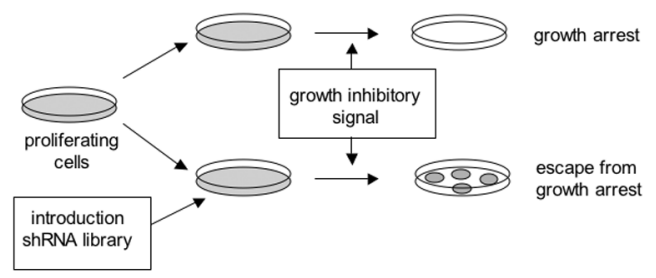

Figure 1. A library of shRNA vectors and its use in mammalian cells. (A) Construction of the NKi shRNA library. From each gene transcript three 19-nucleotide (nt) sequences were designed, and 59-mer oligos were cloned into pRETRO-SUPER (pRS). Three vectors targeting one gene were pooled in a single well of a 96-well plate. From each 96-well plate, high-titer polyclonal virus was produced and used to infect BJ-tsLT cells at $32^{\circ} \mathrm{C}$. After 2 days cells were shifted to $39^{\circ} \mathrm{C}$ and after 3 weeks colonies were isolated and analyzed. $(B)$ Schematic representation of loss-of-function genetic screen to identify shRNA vectors that overcome a proliferation arrest. The shRNA vectors that induce resistance to the growth inhibitory signal will result in continuous proliferation of those cells. The shRNA vectors can readily be recovered from the positively selected cells. 
RNA are separated by a short (9-nucleotide) loop sequence. We have shown previously that this short hairpin precursor transcript is efficiently processed in vivo to yield a gene-specific siRNA-like transcript, causing a gene-specific knockdown of gene expression that lasts as long as the vector is stably expressed (Brummelkamp et al. 2002b). These 59-mers were subsequently annealed and cloned in high throughput fashion into pRETRO-SUPER, a self-inactivating retroviral vector that contains the short hairpin expression cassette (Brummelkamp et al. 2002a). The collection of 24,000 shRNA vectors was cloned in a high-throughput fashion. The bacterial colonies containing one of three shRNA vectors that target the same human transcript for suppression were pooled in a single well of a 96-well plate (Fig. 1A). Using a series of quality control experiments, we estimate that the cloning of the 59-mer oligonucleotides in the retroviral vector was $99 \%$ efficient. By testing a number of pools of three knockdown vectors that target the same gene in transient transfection experiments, we estimate that we obtain at least $70 \%$ inhibition of gene expression for approximately $70 \%$ of the genes in the library (Berns et al. 2004).

\section{ShRNA LIBRARY SCREENING STRATEGIES}

There are several ways in which shRNA libraries can be screened. The most straightforward approach is to generate high-titer retroviral supernatants of (subsets of) the retroviral shRNA vector library and use these supernatants to infect cells of choice, followed by selection of cells with altered phenotype (Fig. 1A). The simplest iteration of such a loss-of-function genetic screen employs mammalian cell systems in which the knockdown vector confers a selective advantage onto the cells harboring the knockdown vector (Fig. 1B). For example, one could induce cancer cells to undergo proliferation arrest by antiproliferative agents, such as TGF- $\beta$ or anticancer drugs. Exposure of cells to these agents will induce proliferation arrest, unless the cells have acquired resistance to the antiproliferative agent through expression of a specific shRNA vector. Such shRNA vectors are positively selected by conferring a growth advantage and can be readily recovered from the proliferating cells by a polymerase chain reaction (PCR)-based strategy (Berns et al. 2004). A similar scheme could be used for agents that induce programmed cell death. The advantage of such a genetic screen is that the identified shRNA is causally linked to the pathway of interest. A drawback is that the performance of such screens is time consuming, as the cells from the primary screen often contain multiple shRNA vectors, making a second-round selection mandatory to confirm the identity of the active shRNA in the screen. We have recently performed the first genetic screen of this type in human cells to identify novel components of the p53 tumor suppressor pathway (Berns et al. 2004; and see below). In addition, we describe here the validation of a second way to screen shRNA vector libraries, which we have named "siRNA bar code screening" (Brummelkamp and Bernards 2003). This technology allows the identification of both shRNA vectors that are positively selected and those that are negatively selected under selective conditions.

\section{A SCREEN FOR BYPASS OF p53 PROLIFERATION ARREST}

The p53 tumor suppressor pathway is crucial to the maintenance of genome integrity as it transmits both antiproliferative and proapoptotic signals in response to a variety of stress signals (Sherr 1998, 2001). Consequently, the $p 53$ gene is mutated in more than half of all human cancers. In addition, the other major components of the p53 pathway such as $p 19^{A R F}$ in mouse ( $p 14^{A R F}$ in man) and MDM2 (HDM2 in man) are mutated in many forms of cancer. This raises the possibility that additional, yet to be discovered, components of this pathway may also be major players in oncogenesis. We therefore focused on the p53 pathway for the identification of novel components through large-scale loss of function genetic screens using the collection of shRNA vectors described above.

To identify cDNAs that allow bypass of the p53-dependent senescence response, we have previously used a "conditionally immortalized" mouse fibroblast cell line (Shvarts et al. 2002). This cell line harbors a temperaturesensitive allele of SV40 large T antigen (tsLT), which inactivates both $\mathrm{pRb}$ and $\mathrm{p} 53$ at $32^{\circ} \mathrm{C}$, but not at $39^{\circ} \mathrm{C}$. As a result, these cells proliferate at $32^{\circ} \mathrm{C}$, but enter into a p53dependent and synchronous proliferation arrest when shifted to $39^{\circ} \mathrm{C}$ (Shvarts et al. 2002). Because the shRNA library that we constructed targets human genes for suppression, we first generated a human equivalent of these "conditionally immortalized" mouse fibroblasts. In primary human fibroblasts, the ability to proliferate indefinitely is not only determined by the presence of $\mathrm{p} 53$, but also by the retinoblastoma protein $\mathrm{pRB}$ and by expression of the telomerase catalytic subunit TERT (de Lange 1998; Lundberg et al. 2000). To generate a conditionally immortalized human fibroblast cell line, we used primary foreskin (BJ) fibroblasts (Bodnar et al. 1998). These cells were first modified by retroviral transduction to express the murine ecotropic receptor and the TERT enzyme. We then introduced tsLT into these BJ-TERT fibroblasts (Lee et al. 1995), yielding BJ-TERT-tsLT cells. These cells proliferate when grown at $32^{\circ} \mathrm{C}$, but enter into a synchronous proliferation arrest after shift to the nonpermissive temperature $\left(39^{\circ} \mathrm{C}\right)$. We found that the growth arrest of these BJ cells is primarily dependent on the p53 tumor suppressor gene, indicating that these cells can be used to identify novel components of the p53 pathway (Berns et al. 2004).

To screen for novel components of the p53 pathway, we isolated polyclonal plasmid DNA from each of the eighty-three 96-well plates of shRNA vectors that together constitute the shRNA library and transfected these pools of vectors into the Phoenix packaging cell line to generate high-titer retroviral supernatants. These retroviruses were then used to infect BJ-TERT-tsLT cells in six-well plates at $32^{\circ} \mathrm{C}$. After 2 days, the cells were shifted to $39^{\circ} \mathrm{C}$ and monitored for the appearance of proliferating colonies over a period of 3 weeks. In a first screen, we identified six genes that allow outgrowth of colonies at $39^{\circ} \mathrm{C}$. One of these six genes was p53 itself, which underscores the quality of the shRNA library. In addition, we found that shRNAs against RPS6KA6 (ribo- 
somal S6 kinase 4, RSK4), HTATIP (histone acetyl transferase TIP60), HDAC4 (histone deacetylase 4), KIAA0828 (a putative S-adenosyl-L-homocysteine hydrolase, SAH3), and CCT2 (T-complex protein 1, b subunit) prevented the p53-dependent growth arrest in the BJ-TERT-tsLT fibroblasts. Subsequent experiments, reported elsewhere (Berns et al. 2004), showed that the identified shRNA constructs also mediated escape from a proliferation arrest induced by the $p 19^{A R F}$ tumor suppressor, whose ability to induce cell cycle arrest is strictly p53-dependent (Kamijo et al. 1997). Furthermore, we found that each of the identified siRNAs confers resistance to G1 cell cycle arrest induced by DNA damage (also a p53-dependent process). These data indicate that expression of these shRNA vectors primarily allows bypass of the p53 response and raise the possibility that the targeted genes by these shRNAs are tumor suppressor genes, as their loss abrogates several cancer-relevant aspects of p53 function. Moreover, these data demonstrate the feasibility of the "positive selection" approach (outlined in Fig. 1B) to identify shRNA vectors that confer loss-of-function phenotypes in mammalian cells.

\section{SIRNA BAR CODE SCREENS}

The shRNA screen described above is time-consuming in that individual colonies of cells must be isolated and hairpin vectors recovered and tested in second-round selection. We have recently proposed an alternative strategy to rapidly screen complex shRNA vector libraries in a polyclonal format to identify individual vectors that either positively or negatively modulate cellular fitness, a technique that we named "siRNA bar code screens" (Brummelkamp and Bernards 2003). This approach was first applied in bacteria and later in yeast (Hensel et al. 1995; Shoemaker et al. 1996; Giaever et al. 1999; Winzeler et al. 1999) and takes advantage of the fact that each hairpin vector contains a unique molecular identifier: The 19-mer sequence in the vector is gene specific. This "molecular bar code" can be used to follow the relative abundance of individual shRNA vectors in a large population of infected cells. The molecular bar codes can be recovered from a cell population by PCR amplification using vector-derived PCR primers that flank the hairpinencoding DNA sequence. The relative abundance of the shRNA vectors in a cell population (and therefore of their bar code identifier sequences) is influenced by the effect that each knockdown vector has on cellular fitness under the experimental conditions (which will often be a "stress of interest"). Fluorescent labeling of the bar code sequence followed by hybridization to microarrays that contain the gene-specific knockdown bar code oligonucleotides allows one to monitor the relative abundance of each bar-coded DNA fragment in the cell population (Fig. 2) (Brummelkamp and Bernards 2003). Therefore, siRNA bar code screens allow one to detect all genes that have a genetic interaction with a cell population that is exposed to a "stress of interest." As such, this technology allows one to make functional interaction maps of the human genome by being able to efficiently map all genes that modulate a cellular response to a given stress.

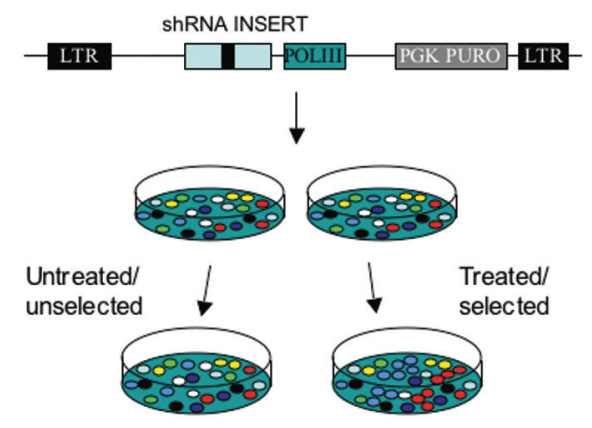

shRNA probes generated by $P C R$, in vitro RNA amplification and fluorescent labeling with Cy3 or Cy5 dyes
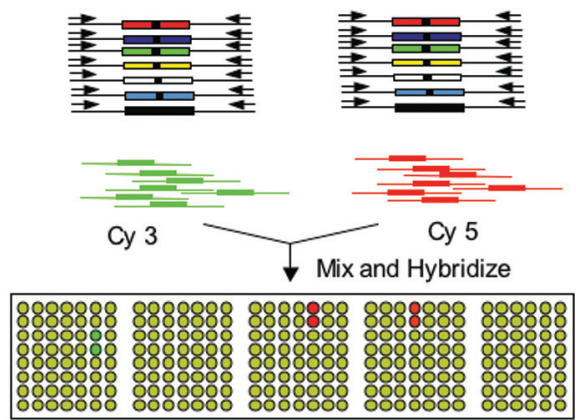

Figure 2. Schematic representation of a bar code screening experiment. Expression of short hairpin RNA (shRNA) molecules in mammalian cells by stably integrated vectors not only creates a gene-specific knockdown phenotype, but also introduces a gene-specific fingerprint (molecular bar code) in cells that express these shRNAs. The 19-base-pair target-gene-specific insert of the shRNA vector is unique in sequence and can be recovered by polymerase chain reaction (PCR) using vector-derived PCR primers that flank the hairpin-encoding DNA sequence. The PCR fragments are amplified by in vitro RNA amplification using the T7 polymerase, whose promoter is present in one of the primer sequences. The abundance of each bar code can be quantified by labeling the RNA fragments with a fluorescent dye, followed by hybridization to a DNA microarray harboring of bar code-complementary DNA fragments. shRNA bar code screens allow the detection of genetic interactions between large sets of genes and almost any biological signal of interest by comparing two cell populations, both of which harbor a collection of shRNA vectors, but only one of which is exposed to a biological signal (e.g., DNA damage, apoptosis-inducing agents, cytotoxic drugs, or inactivation of a tumor suppressor gene). The comparative hybridization of the Cy5- and Cy3-labeled bar-coded RNA fragments allows for the identification of changes (increase or decrease) in the relative abundance of knockdown vectors in response to the stimulus applied.

\section{VALIDATION OF SIRNA BAR CODING USING OLIGONUCLEOTIDE MICROARRAYS}

The siRNA bar code screening technology provides both opportunities and challenges. One of the challenges is that the 19-mer "molecular bar code" sequence contained within the shRNA vector is part of a hairpin structure that has the ability to fold back on itself (Fig. 3A). This could potentially limit hybridization signals that one obtains when using hairpin probes for hybridization to microarrays. The shRNA vector library was constructed using a set of forty-eight thousand 59-mer oligonu- 
cleotides, which include the 19-mer molecular bar code sequences. These 59-mer oligonucleotides provide an attractive source for the generation of siRNA bar code microarrays, as large amounts of these oligonucleotides were left over from the construction of the shRNA library. However, these 59-mers are also self-complementary and might also fold back on themselves rather than hybridize to the Cy-dye-labeled probes to which they are hybridized.

To address whether these hybridization issues pose a problem, we first performed a number of experiments designed to test the relative sensitivity and specificity of hybridization of shRNA probes to DNA microarrays containing the 59-mer oligonucleotide bar code identifiers. Specifically, we asked how efficiently we could identify an arbitrary set of 188 shRNA vectors. To do this, we performed a self-self hybridization with a pool of 188 different 59-mer oligonucleotides used for cloning of the NKi shRNA library. The concentration for each oligonucleotide in the mixture was $200 \mathrm{pg}$ and the mixtures were labeled with either $\mathrm{Cy} 3$ or $\mathrm{Cy} 5$ followed by hybridization to DNA containing the complementary 59-mer oligonucleotides from the NKi shRNA library. As depicted in
Figure $3 \mathrm{~B}$, the signal intensities of the spots on the array corresponding to the set of 188 vectors were significantly different from the remainder of the spots (which contained unrelated 59-mer oligonucleotides). The signal intensity from the specific sequences was $\sim 100$-fold higher than those from the spots containing noncomplementary 59-mer oligonucleotides. This experiment shows that hairpin probes can be used for hybridization and they do so with good specificity and high signal intensity.

Next, we asked whether the hybridization signals on the array accurately reflect the concentration of the molecular bar code probes in the population. To test this we performed a titration experiment using a pool of 1344 different 59-mer oligonucleotides. In one sample, the amount of the oligonucleotides was held constant at 200 pg; in the other sample the amount of the oligonucleotides was reduced in twofold dilution steps $(200 \mathrm{pg}, 100 \mathrm{pg}, 50$ $\mathrm{pg}, 25 \mathrm{pg}, 12.5 \mathrm{pg}, 6.25 \mathrm{pg}$, and $3.12 \mathrm{pg}$ ) for groups of 192 oligonucleotides. The samples were labeled with $\mathrm{Cy} 3$ or Cy5, respectively, combined, and hybridized to a microarray containing all 23,742 oligonucleotides (representing the complete shRNA library). As can be seen in the representative section of the microarray (Fig. 3C), we
A.

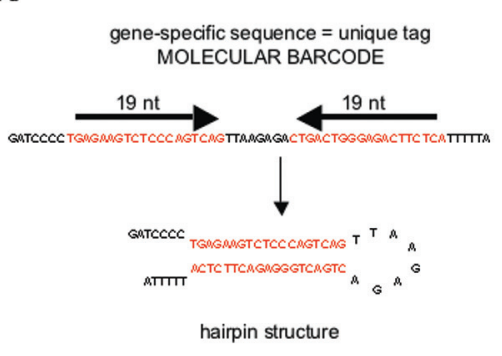

c.

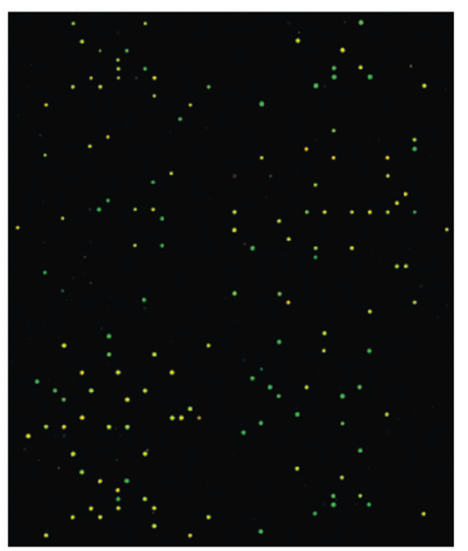

B.

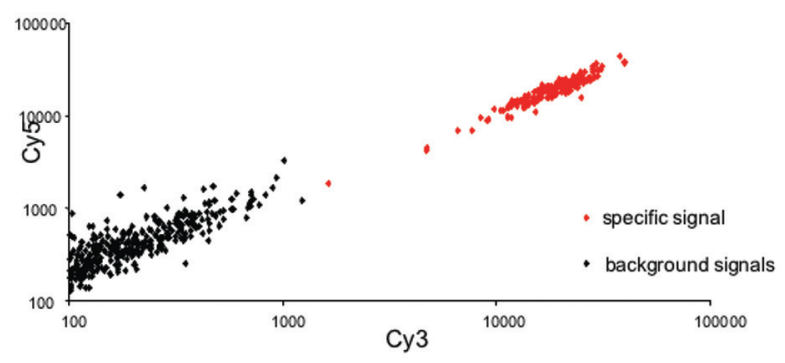

D.

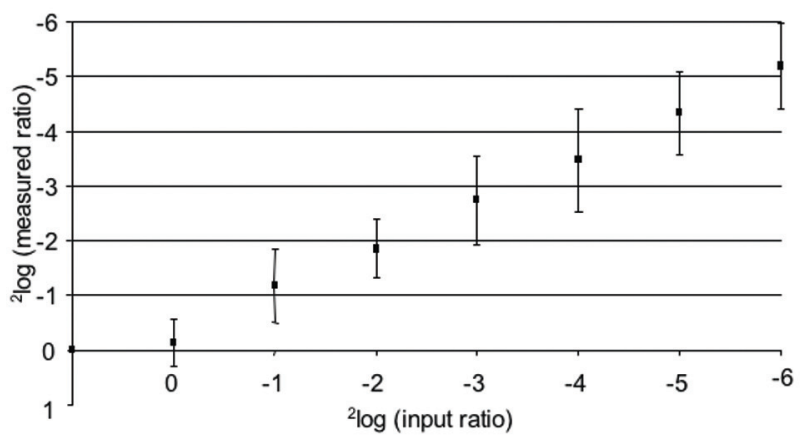

Figure 3. Microarray analysis of shRNA library bar codes. (A) Schematic representation of the 59-mer oligonucleotide, including the 19-mer gene-specific sequences, which serves as a molecular bar code sequence. The 59-mer oligonucleotide is part of the shRNA hairpin structure and has the ability to fold back on itself. $(B)$ Self-self hybridization of a subset of 59-mer oligonucleotides as depicted in $A$ on a microarray containing the complementary 59-mer sequences. A pool of 188 different 59-mer oligonucleotides was used with $200 \mathrm{pg}$ of each oligonucleotide. Depicted in red are the hybridization signals obtained from the specific complementary oligonucleotides. Depicted in black are 376 nonhomologous oligonucleotides representing nonspecific background hybridization. The average intensity of specific hybridization compared to average background hybridization is more than 100-fold. (C) A representative section of a microarray hybridized with a mixture of 1344 different 59-mer oligonucleotides. Hybridizations were performed with two mixtures: a Cy3-labeled mixture in which a constant amount of $200 \mathrm{pg}$ of each oligonucleotide was present and a Cy5-labeled mixture in which the amount was varied (3.1-200 pg) for sets of 192 different oligonucleotides. $(D)$ Plot showing the normalized data from the titration study described in $C$. For each set of 192 oligonucleotides the average ratio is calculated plus the standard deviation. Depicted are the ${ }^{2} \log$ from the input ratio (1:1 to $1: 64$ ratios depicted as 0 to -6$)$ and the ${ }^{2} \log$ from the measured ratio. 
obtain specific hybridization with high signal intensities. From the 1344 spots, representing the composition of the probe mixture, we calculated the average ratio and standard deviation for all the different groups of 192 oligonucleotides reflecting a 1:1 to $1: 64$ dilution series. This ratio was dependent on the relative abundance of the oligonucleotides in the mixture and a twofold change was detected (Fig. 3D). Most importantly, there is a linear correlation between the experimentally determined average ratio of the groups of 192 oligonucleotides compared to the input ratio. The hybridization intensities for the serially diluted oligonucleotides was reflected in linear fashion over the 64-fold dilution range tested (Fig. 3D). This experiment demonstrates the feasibility of using DNA microarrays to determine the relatively abundance of shRNA vectors in a large population and shows that the sensitivity of the approach is sufficient to detect relatively small differences (two- to fourfold) in the presence of shRNA abundance within a complex mixture of shRNAs.

Next we tested whether our experimental approach allowed us to use shRNA probes recovered from cell populations expressing large numbers of shRNA constructs. We generated shRNA cassettes by PCR from cells infected with a pool of 1700 shRNA vectors using vector-derived primers of which one was fused to the T7 promoter sequence. This was used in an in vitro RNA amplification reaction followed by labeling of the in vitro generated RNA with Cy5 dye. The RNA probes were used in a hybridization reaction together with the 1344 59-mer oligonucleotide mixture labeled with Cy3. Figure 4A shows a representative section of a microarray containing all 23,742 oligonucleotides representing the complete NKi shRNA library. The RNA probes generate high signal intensities with virtually no cross-hybridization to noncomplementary spots, similar to what is seen with the 59-mer oligonucleotide probes. In this hybridization experiment, the signal intensity for the RNA probes is more variable than that of the 59-mer oligonucleotides. These differences reflect the variation in abundance of the different shRNA vectors present in the mixture compared to the constant amount of 59-mer oligonucleotides (200 pg each). This experiment demonstrates that shRNA probes generated by PCR followed by linear RNA amplification are suitable for use in microarray hybridization experiments.

Next we tested whether our experimental approach allowed us to use shRNA probes from a large population of shRNA vectors in microarray hybridizations. We generated shRNA probes from two pools of each 5472 shRNA plasmids by PCR followed by in vitro linear RNA amplification. This RNA was subsequently labeled with $\mathrm{Cy} 3$ or Cy5 dye and used for hybridization to the DNA microarray. As can be seen in Figure 4, we obtain strong hybridization signals for both $\mathrm{Cy} 3$ - and $\mathrm{Cy} 5$-labeled probe mixtures. The spots harboring oligonucleotides not present in either probe pool hybridize at background levels, emphasizing again the specificity of the hybridization of the bar code oligonucleotides. This experiment demonstrates the feasibility of the use of DNA microarray hy-
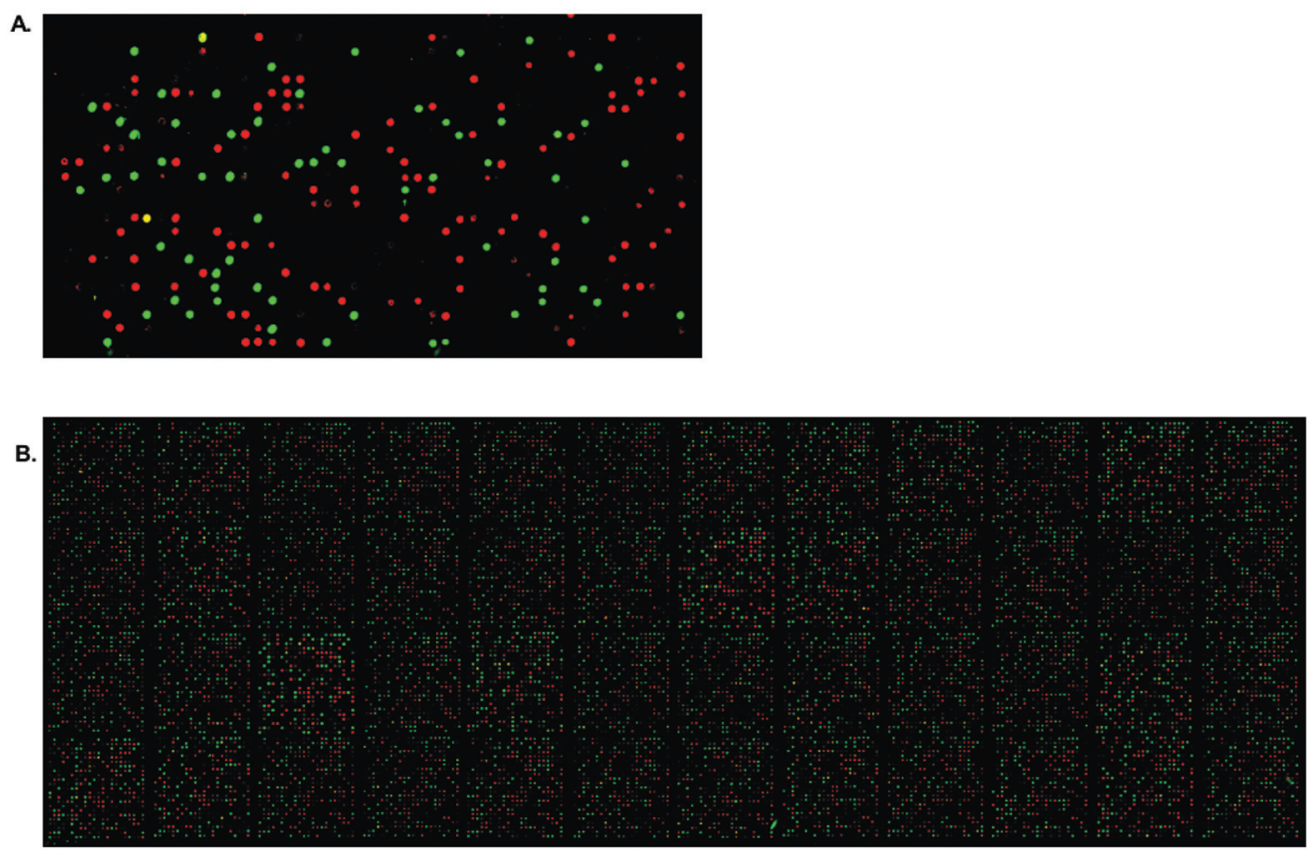

Figure 4. Validation of the specific hybridization with RNA bar code probe mixtures. $(A)$ A representative fraction of a microarray for the comparison of hybridization with labeled 59-mer oligonucleotides (Cy3, green) and labeled amplified RNA probes containing the hairpin sequences $(\mathrm{Cy} 5, \mathrm{red})$. Both probes generate high signal intensities with virtually no cross-hybridization on noncomplementary spots. In this hybridization experiment, the signal intensity for the RNA probes has more variation than the 59-mer oligonucleotides, reflecting the variation in abundance of the different shRNA vectors present compared to the constant amount of 59mer oligonucleotides (200 pg each). (B) Representation of a DNA microarray containing 23,742 oligonucleotides (representing the complete NKi shRNA library) hybridized with two different shRNA probe mixtures containing two different groups of 5000 different shRNA probes labeled Cy3 (green) or Cy5 (red). 
bridizations in conjunction with the full complexity of the shRNA library. It also underscores the sensitivity in this system to detect absolute differences in two large populations of shRNA vectors. This result, in combination with the experiments described above, demonstrates the application of shRNA bar code screens in the detection of small differences in shRNA abundance in parallel screening of large populations of shRNA vectors.

\section{VALIDATION OF BAR CODE SCREENING IN A CELL SYSTEM}

As a last validation of the bar code screening concept, we asked whether we could also use the bar coding technology to identify components in the p53 pathway using the conditionally immortalized human fibroblasts (BJTERT-tsLT cells) described in more detail by Berns et al. (2004). The design of this experiment is depicted in Figure 5A. We generated a set of 1900 different shRNA vectors targeting 638 different genes. We used this set of vectors to infect the BJ-TERT-tsLT cells, selected for the presence of integrated retroviruses with puromycin. After this, the cells were split at low density and either grown at $32^{\circ} \mathrm{C}$ (permissive temperature) or at $39^{\circ} \mathrm{C}$ (restrictive temperature). The cells grown at $32^{\circ} \mathrm{C}$ continued to pro- liferate and genomic DNA was isolated when cells reached confluency, after about 8 days. In contrast, the cells cultured at $39^{\circ} \mathrm{C}$ underwent proliferation arrest and only those cells that harbored a shRNA vector that conferred resistance to the $\mathrm{p} 53$-dependent arrest continued to proliferate. As a result, the shRNA vectors associated with the capacity to proliferate at $39^{\circ} \mathrm{C}$ will be selectively enriched in the population. After 22 days, genomic DNA was isolated from the cultures at $39^{\circ} \mathrm{C}$ and the relative abundance of shRNA vectors was analyzed on DNA microarrays containing all 1900 shRNA sequences (Fig. 5B). As expected the majority of the shRNA vectors was neither positively or negatively selected (yellow spots) and had a fluorescence intensity ratio ( ${ }^{2} \log$ ratio) varied from -1 to 1 (Fig. 5C). However, some shRNA vectors were enriched in the population at $39^{\circ} \mathrm{C}$ (red spots). Upon further analysis (multiple independent temperature-shift experiments and array hybridizations), two shRNA vectors were identified that were consistently enriched in the population at $39^{\circ} \mathrm{C}$ (Fig. 5C,D). Both shRNA vectors target the cell cycle inhibitor $\mathrm{p} 21^{\text {cip } 1}$ (CDKNA1) and were shown previously to suppress $\mathrm{p} 21^{\mathrm{cip} 1}$ expression and override the p53-induced cell cycle arrest in the BJ fibroblasts (Berns et al. 2004). The third shRNA vector against p $21^{\text {cip } 1}$ was not enriched at $39^{\circ} \mathrm{C}$ and was subsequently

A. Infect with shRNA vectors

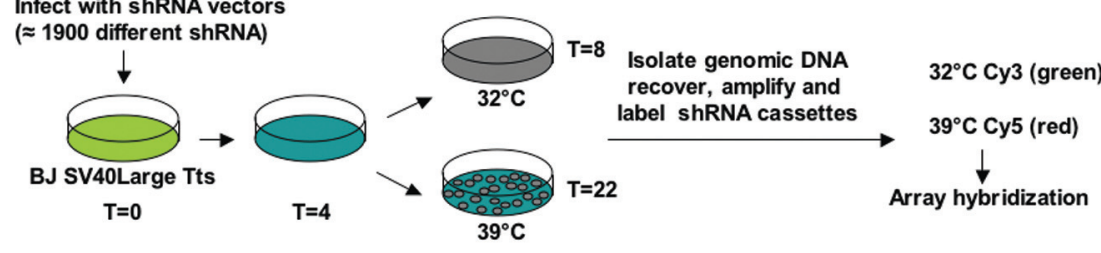

B.

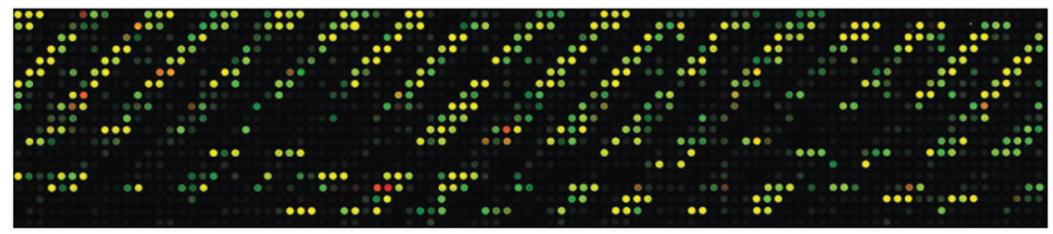

C.

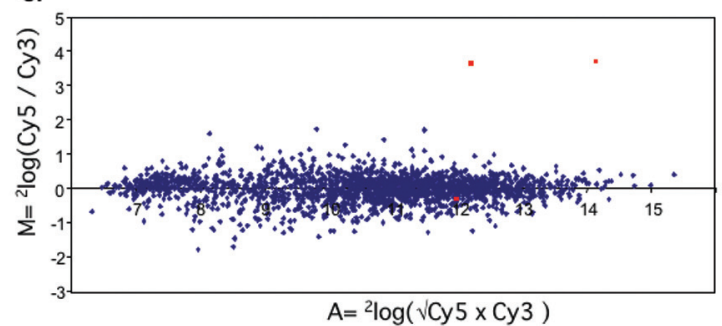

D.

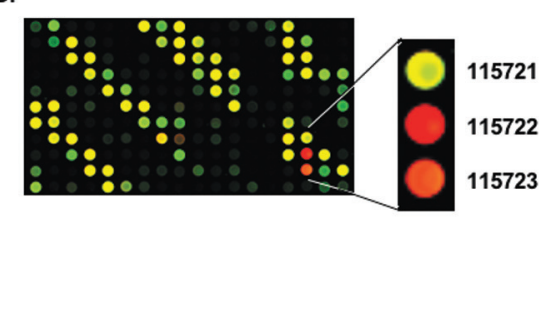

Figure 5. Validation of bar code screening in the BJ-TERT-tsLT system. (A) Schematic representation of the experimental setup of the bar code screen in BJ-TERT-tsLT cells. BJ-TERT-tsLT cells were infected with a retroviral supernatant containing 1900 shRNA vectors. Cells were selected for successful retroviral transduction and temperature shifted to induce a senescence-like arrest and used for isolation of the genomic DNA at time $(T)=8$ days at $32^{\circ} \mathrm{C}$ or at time $(T)=22$ days at $39^{\circ} \mathrm{C}$. The shRNA cassettes were recovered by PCR, amplified by linear RNA amplification, and labeled with $\mathrm{Cy} 3\left(32^{\circ} \mathrm{C}\right)$ or $\mathrm{Cy} 5\left(39^{\circ} \mathrm{C}\right)$. (B) A representative section of the microarray hybridization of shRNA fragments isolated from the experiment described in $A$. (C) Analysis of the relative abundance of shRNAs in BJ-TERT-tsLT cells recovered from cells grown at $32^{\circ} \mathrm{C}$ or at $39^{\circ} \mathrm{C}$. Data are normalized and depicted as $M$, the ${ }^{2} \log ($ ratio $\mathrm{Cy} 5 / \mathrm{Cy} 3)$ versus $A$, the ${ }^{2} \log (\sqrt{ }$ intensity $\mathrm{Cy} 3 \times \mathrm{Cy} 5)$. The data are the average of three independent experiments performed in duplicate and with reversed color. The three red spots indicate shRNA vectors targeting the p21 gene. (D) Cutout of the section of the DNA microarray containing the three shRNAs for $\mathrm{p} 21$. The shRNAs 115722 and 15723 are specifically enriched at $39^{\circ} \mathrm{C}$ whereas 115721 is not affected. Both shRNAs 115722 and 115723 are active shRNAs against p21, whereas 115721 is inactive. 
shown to be inactive in downregulation of $\mathrm{p} 21^{\mathrm{cip} 1} \mathrm{ex}-$ pression. Together these data show that the siRNA bar code screening can be used to rapidly identify individual shRNA vectors that modulate cellular responses in a large library of vectors. The identification of $\mathrm{p} 21^{\text {cip } 1}$ in this screen also validates the application of the bar code screening method in a relevant biological setting in which the relative enrichment of shRNA vectors is influenced by many biological parameters.

\section{CONCLUSIONS}

We describe here two approaches to identify shRNA vectors that confer loss-of-function phenotypes in mammalian cells. The siRNA bar code screening technology presented and validated here can be very helpful in the identification of novel anticancer drug targets. For instance, a potentially useful application of siRNA bar code screens is that they facilitate the identification of synthetic lethal interactions (a combination of two nonlethal mutations that together result in cell death). The identification of genes that are specifically toxic in cells having cancer-specific mutations provides an opportunity to make new generations of anticancer drugs that are more tumor-specific and have fewer side effects.

\section{ACKNOWLEDGMENTS}

We thank Stephen Friend, Peter Linsley, Guy Cavet, Wei Ge, and Julian Downward for their support of the shRNA library project. This work was supported by grants from the Netherlands Genomics Initiative/Netherlands Organisation for Scientific Research (NWO), Cancer Research UK, the Centre for Biomedical Genetics (CBG), and the Dutch Cancer Society (KWF).

\section{REFERENCES}

Ashrafi K., Chang F.Y., Watts J.L., Fraser A.G., Kamath R.S., Ahringer J., and Ruvkun G. 2003. Genome-wide RNAi analysis of Caenorhabditis elegans fat regulatory genes. Nature 421: 268.

Aza-Blanc P., Cooper C.L., Wagner K., Batalov S., Deveraux Q.L., and Cooke M.P. 2003. Identification of modulators of TRAIL-induced apoptosis via RNAi-based phenotypic screening. Mol. Cell 12: 627.

Berns K., Hijmans E.M., Mullenders J., Brummelkamp T.R., Velds A., Heimerikx M., Kerkhoven R.M., Madiredjo M., Nijkamp W., Weigelt B., Agami R., Ge W., Cavet G., Linsley P.S., Beijersbergen R.L., and Bernards R. 2004. A large-scale RNAi screen in human cells identifies new components of the p53 pathway. Nature 428: 431.

Bodnar A.G., Ouellette M., Frolkis M., Holt S.E., Chiu C.P., Morin G.B., Harley C.B., Shay J.W., Lichtsteiner S., and Wright W.E. 1998. Extension of life-span by introduction of telomerase into normal human cells. Science 279: 349.

Brummelkamp T.R. and Bernards R. 2003. New tools for functional mammalian cancer genetics. Nat. Rev. Cancer 3: 781.

Brummelkamp T.R., Bernards R., and Agami R. 2002a. Stable suppression of tumorigenicity by virus-mediated RNA interference. Cancer Cell 2: 243.

- 2002b. A system for stable expression of short interfering RNAs in mammalian cells. Science 296: 550.

Brummelkamp T.R., Nijman S.M., Dirac A.M., and Bernards R. 2003. Loss of the cylindromatosis tumour suppressor inhibits apoptosis by activating NF-kappaB. Nature 424: 797. de Lange T. 1998. Telomeres and senescence: Ending the debate. Science 279: 334.

Elbashir S.M., Harborth J., Lendeckel W., Yalcin A., Weber K., and Tuschl T. 2001. Duplexes of 21-nucleotide RNAs mediate RNA interference in cultured mammalian cells. Nature 411: 494.

Fire A., Xu S., Montgomery M.K., Kostas S.A., Driver S.E., and Mello C.C. 1998. Potent and specific genetic interference by double-stranded RNA in Caenorhabditis elegans. Nature 391: 806

Giaever G., Shoemaker D.D., Jones T.W., Liang H., Winzeler E.A., Astromoff A., and Davis R.W. 1999. Genomic profiling of drug sensitivities via induced haploinsufficiency. Nat. Genet. 21: 278.

Hensel M., Shea J.E., Gleeson C., Jones M.D., Dalton E., and Holden D.W. 1995. Simultaneous identification of bacterial virulence genes by negative selection. Science 269: 400 .

Kamath R.S., Fraser A.G., Dong Y., Poulin G., Durbin R., Gotta M., Kanapin A., Le Bot N., Moreno S., Sohrmann M., Welchman D.P., Zipperlen P., and Ahringer J. 2003. Systematic functional analysis of the Caenorhabditis elegans genome using RNAi. Nature 421: 231.

Kamijo T., Zindy F., Roussel M.F., Quelle D.E., Downing J.R., Ashmun R.A., Grosveld G., and Sherr C.J. 1997. Tumor suppression at the mouse INK4a locus mediated by the alternative reading frame product p19ARF. Cell 91: 649 .

Lee G.H., Ogawa K., and Drinkwater N.R. 1995. Conditional transformation of mouse liver epithelial cells. An in vitro model for analysis of genetic events in hepatocarcinogenesis. Am. J. Pathol. 147: 1811.

Lum L., Yao S., Mozer B., Rovescalli A., Von Kessler D., Nirenberg M., and Beachy P.A. 2003. Identification of Hedgehog pathway components by RNAi in Drosophila cultured cells. Science 299: 2039.

Lundberg A.S., Hahn W.C., Gupta P., and Weinberg R.A. 2000. Genes involved in senescence and immortalization. Curr. Opin. Cell Biol. 12: 705.

Miyagishi M. and Taira K. 2002. U6 promoter-driven siRNAs with four uridine $3^{\prime}$ overhangs efficiently suppress targeted gene expression in mammalian cells. Nat. Biotechnol. 20: 497.

Paddison P.J., Caudy A.A., Bernstein E., Hannon G.J., and Conklin D.S. 2002. Short hairpin RNAs (shRNAs) induce sequencespecific silencing in mammalian cells. Genes Dev. 16: 948.

Sherr C.J. 1998. Tumor surveillance via the ARF-p53 pathway. Genes Dev. 12: 2984.

- 2001. The ink4a/arf network in tumour suppression. Nat. Rev. Mol. Cell Biol. 2: 731.

Shoemaker D.D., Lashkari D.A., Morris D., Mittmann M., and Davis R.W. 1996. Quantitative phenotypic analysis of yeast deletion mutants using a highly parallel molecular bar-coding strategy. Nat. Genet. 14: 450 .

Shvarts A., Brummelkamp T.R., Scheeren F., Koh E.Y., Daley G.Q., Spits H., and Bernards R. 2002. A senescence rescue screen identifies BCL6 as an inhibitor of anti-proliferative p19(ARF)-p53 signaling. Genes Dev. 16: 681.

Stark G.R., Kerr I.M., Williams B.R., Silverman R.H., and Schreiber R.D. 1998. How cells respond to interferons. Annu. Rev. Biochem. 67: 227.

Sui G., Soohoo C., Affar el B., Gay F., Shi Y., and Forrester W.C. 2002. A DNA vector-based RNAi technology to suppress gene expression in mammalian cells. Proc. Natl. Acad. Sci. 99: 5515 .

Winzeler E.A., Shoemaker D.D., Astromoff A., Liang H., Anderson K., Andre B., Bangham R., Benito R., Boeke J.D., Bussey H., Chu A.M., Connelly C., Davis K., Dietrich F., Dow S.W., El Bakkoury M., Foury F., Friend S.H., Gentalen E., Giaever G., Hegemann J.H., Jones T., Laub M., Liao H., and Davis R.W. 1999. Functional characterization of the $S$. cerevisiae genome by gene deletion and parallel analysis. Science 285: 901.

Yu J.Y., DeRuiter S.L., and Turner D.L. 2002. RNA interference by expression of short-interfering RNAs and hairpin RNAs in mammalian cells. Proc. Natl. Acad. Sci. 99: 6047. 


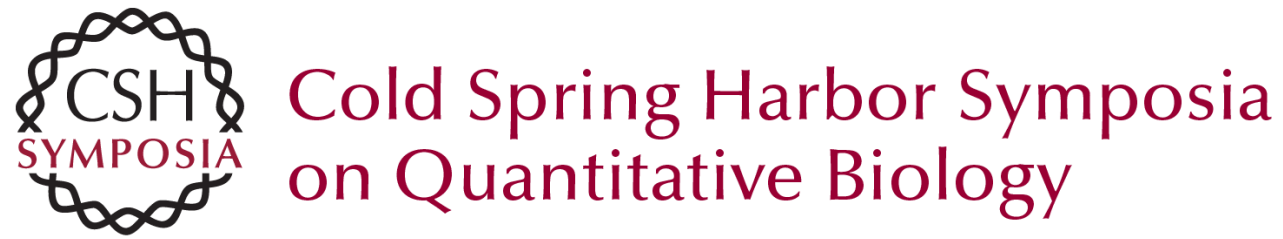

\section{Functional Identification of Cancer-relevant Genes through Large-Scale RNA Interference Screens in Mammalian Cells}

T.R. BRUMMELKAMP, K. BERNS, E.M. HIJMANS, et al.

Cold Spring Harb Symp Quant Biol 2004 69: 439-446

Access the most recent version at doi:10.1101/sqb.2004.69.439

References This article cites 28 articles, 11 of which can be accessed free at: http://symposium.cshlp.org/content/69/439.full.html\#ref-list-1

License

Email Alerting Receive free email alerts when new articles cite this article - sign up in Service the box at the top right corner of the article or click here.

To subscribe to Cold Spring Harbor Symposia on Quantitative Biology go to: 